\title{
Heme Oxygenase-1 Mediates Oxidative Stress and Apoptosis in Coxsackievirus B3-Induced Myocarditis
}

\author{
Oana N. Ursu Martina Sauter Nicole Ettischer Reinhard Kandolf Karin Klingel \\ Institute for Pathology and Neuropathology, Department of Molecular Pathology, University Hospital \\ Tübingen, Tübingen, Germany
}

\author{
Key Words \\ Myocarditis • Heme oxygenase- 1 - Free radicals • Apoptosis $\bullet$ Iron
}

\begin{abstract}
Background: Heme oxygenase-1 (HO-1), which is suggested to play a role in defending the organism against oxidative stress-mediated injuries, can be induced by diverse factors including viruses and iron. As coxsackievirus B3 (CVB3)-infected SWR/J mice susceptible for chronic myocarditis were found to have a significant iron incorporation and $\mathrm{HO}-1$ upregulation in the myocardium, we aimed to investigate the molecular interplay between $\mathrm{HO}-1$ expression and iron homeostasis in the outcome of viral myocarditis. Methods and Results: In susceptible SWR/J mice, but not in resistant C57BL/6 mice, we observed at later stages of CVB3 myocarditis significant iron deposits in macrophages and also in cardiomyocytes, which were spatially associated with oxidative stress, upregulation of $\mathrm{HO}-1$ and caspase- 3 activation. $\mathrm{HO}-1$, which is also expressed in cultivated RAW 264.7 macrophages upon incubation with iron and/or CVB3, could be downregulated by inhibition of NO/iNOS using L-NAME. Moreover, specific inhibition of HO-1 by tin mesoporphyrin revealed a suppression of superoxide production in iron and/ or CVB3-treated macrophages. The molecular relationship of HO-1 and caspase-3 activation was proven by downregulation with HO-1 siRNA in iron- and/or CVB3-treated cultivated cells. Importantly, iron was found to increase viral replication in vitro. Conclusion: These results indicate that $\mathrm{HO}-1$ induces a paracrine signalling in macrophages via reactive oxygen species production, mediating apoptosis of heart muscle cells at later stages of myocarditis. Notably, in genetically susceptible mice iron potentiates the detrimental effects of CVB3 by the NO/ HO-1 pathway, thus increasing cardiac pathogenicity.
\end{abstract}

Copyright (C) 2014 S. Karger AG, Basel 


\section{Cellular Physiology and Biochemistry}

Cell Physiol Biochem 2014;33:52-66

DOI: 10.1159/000356649

02, 2014

\section{Introduction}

Coxsackieviruses of group B are members of the Picornaviridae family which have been identified as major cause of acute and chronic forms of myocarditis [1]. In case of an ineffective host immune response, these enteroviruses may persist beyond the acute phase of the infection and induce an ongoing inflammation which may lead to dilated cardiomyopathy many years after the initial infection [2]. Coxsackieviruses, including CVB3, affect the cardiomyocytes directly as a consequence of viral replication, but also elicit immune cell infiltrates determining the different outcome of the inflammatory heart disease at later stages of the disease [3] . One important cell type which is decisive in the early antiviral innate immune response are macrophages which were found to define the susceptibility to CVB3 myocarditis via differential cytokine expression [4]. On the other hand, macrophages express inducible nitric oxide synthase (iNOS), which was found to contribute significantly to ongoing damage by nitric oxide (NO) production at later stages of CVB3 infection [5]. NO is a well known mediator for the generation of reactive oxygen species (ROS) and reactive nitrogen species (RNS), which are responsible for the generation of oxidative stress in the inflamed myocardium [6]. Also, CVB3 replication in cardiomyocytes is associated with the production of ROS in these cells [7]. Intracellular ROS levels are generally controlled by different antioxidant defense mechanisms, however, ROS overproduction may result in detrimental intracellular disturbances. One important molecule in the cellular response to stress/oxidant stimuli is heme oxygenase- 1 (HO-1), the inducible isoform of $\mathrm{HO}$, which catalyzes the rate-limiting step of heme oxidation to biliverdin, carbon monoxide, and free ferrous iron [8]. HO-1, the heat shock/stress cognate of the heat shock protein 32 family of proteins, is expressed in response to a variety of stimuli that are associated with oxidative stress and inflammation [9]. Also, HO-1 mRNA and protein expression were found to be increased by intracellular iron overload [10]. Iron is described to improve cardiac function in patients with chronic heart failure (CHF) that are prone to develop iron deficiency [11], however it may also be cardiotoxic under overload conditions [12]. Ironoverload cardiomyopathy is an important cause of heart failure and involves susceptibility to arrhythmias and a late-stage dilated cardiomyopathy. At later stages of this disease, the disturbed iron metabolism in myocytes induces remodelling processes of the heart, features which are also observed as a consequence of enterovirus-induced chronic myocarditis [13]. Interestingly, both CVB3 and iron are known to enhance ROS production. However, there are no data available investigating the molecular interplay between enteroviral infection and iron metabolism in the heart. Thus, the aim of this study was to study the interactions of enterovirus replication, ROS production, iron overload and HO-1 expression in relation to the outcome of myocarditis in CVB3-infected mice.

\section{Materials and Methods}

\section{Mice and infection}

SWR/J and C57BL/6 mice, originally purchased from Jackson Laboratories (Bar Harbor, ME), were kept under specific pathogen-free conditions at the animal facilities of the Department of Molecular Pathology, University Hospital Tübingen, and experiments were conducted according to the German law regarding animal protection (permit no. PA 1/08). At the age of 4 to 5 weeks, mice were intraperitoneally inoculated with $10^{5}$ plaque-forming units of purified CVB3 as previously described [14]. Animals of each strain $(\mathrm{n} \geq 5)$ were sacrificed at days 4, 8, 12 and 28 post infection (p.i.) and hearts, spleen and serum were collected for analysis. For histology, parts of the collected organs were fixed in $4 \%$ paraformaldehyde and embedded in paraffin. For quantitative analyses, parts of the organs were snap-frozen and stored at $-80^{\circ} \mathrm{C}$.

Virus

CVB3 used in this study was derived from the infectious cDNA copy of the cardiotropic Nancy strain and virus stocks and plaque assays were performed as previously described [14]. 


\section{Cellular Physiology and Biochemistry}

Cell Physiol Biochem 2014;33:52-66

DOI: $10.1159 / 000356649$

2, 2014

C) 2014 S. Karger AG, Basel

www.karger.com/cpb

Histology and immunohistochemical staining

Paraffin-embedded hearts were cut in 5- $\mu$ m-thick tissue sections and stained with hematoxylin and eosin to assess myocardial injury and inflammation. For evaluation of fibrosis, Masson trichrome staining was applied while iron deposits were detected by Prussian blue staining. For immunohistochemistry, tissue sections were incubated for 1 hour at $25^{\circ} \mathrm{C}$ with rabbit anti-mouse antibodies recognizing murine HO-1 (1:100, Abcam, UK), cleaved caspase-3 (1:400, Cell Signaling Technology, Danvers, MA, USA). Controls using normal rabbit serum were run to exclude nonspecific staining. Slides were processed using streptABComplex HRP (Dako, Hamburg, Germany) and 3,3` diaminobenzidine (Biozol, Eching, Germany) as substrate. Visualization of CVB3 VP1 protein was done by incubating deparaffinized tissue sections with a monoclonal antibody specific for CVB3 VP1 (1:400, Mediagnost, Reutlingen, Germany) for 1hour at room temperature. Subsequently, the sections were processed with the MaxHomo Mouse on Mouse Polymer HRP Detection Kit (MaxVision, Washington, USA) followed by AEC substrate (Linaris, Dossenheim, Germany). The tissue sections were counterstained with hematoxylin.

\section{Quantitative real- time RT-PCR}

Murine heart tissues were lysed in Trifast (Peqlab, Erlangen, Germany) according to the manufacturer's instructions, and the integrity and concentration of the total RNA was assessed by agarose gel electrophoresis and by photospectrometry, respectively. RNA (200 ng) was used to perform one step quantitative real-time reverse-transcription PCR (TaqMan One-Step RT-PCR Master Mix Reagents Kit, Applied Biosystems, Foster City, USA) at the appropriate annealing temperature for 40 cycles. Data analysis was performed as relative quantification in relation to the expression of the housekeeping gene hypoxanthin-guanin-phosphoribosyltransferase (HPRT) as internal standard. The absolute number of CVB3 viral genomes was determined in relation to an external virus standard using PanEntero-primers and probes. Specific primers and probes were purchased from MWG Biotech (Ebersberg, Germany).

\section{In situ hybridization (ISH)}

CVB3 positive-strand genomic RNA in tissues was detected using single-stranded ${ }^{35} \mathrm{~S}$-labeled RNA probes as previously described [14]. For detection of iNOS mRNA in tissues, ${ }^{35} \mathrm{~S}$-labeled anti-sense and sense iNOS RNA probes were synthesized as previously described [5]. For detection of HO-1 mRNA, anti-sense and sense RNA probes were synthesized based on a mHO-1 plasmid (imaGenes, Berlin, Germany). Pretreatment, hybridization, and washing procedures of dewaxed $5 \mu \mathrm{m}$ paraffin tissue sections were performed as previously described [14]. Slide preparations were subjected to autoradiography, exposed for 3 weeks at $4^{\circ} \mathrm{C}$, and counterstained with haematoxylin/eosin

\section{Dihydroethidium staining (DHE)}

In situ production of superoxide anion was assessed in $5 \mu \mathrm{m}$ frozen heart tissue sections with the oxidative fluorescent dye dihydroethidium (DHE, Invitrogen, Karlsruhe, Germany). Sections were fixed for 10 minutes in cold acetone, washed in PBS and incubated in DHE (final concentration of 5 $\mu \mathrm{mol} / \mathrm{L}$ ) for 15 minutes at $37^{\circ} \mathrm{C}$ and counterstained with DAPI. Fluorescence images were obtained with a Zeiss Axiovert 2000 microscope. For superoxide detection in cells, live-imaging experiments were performed in irontreated and CVB3-infected coculture of HeLa cells and RAW 264.7 macrophages. In order to differentiate between RAW 264.7 macrophages and HeLa cells, macrophages were labeled with CellTracker Green (Lonza, Germany) before DHE labeling. For the live-imaging, cells were kept in an incubation chamber on the microscope at $37^{\circ} \mathrm{C}, 5 \% \mathrm{CO}_{2}$ and in humid atmosphere.

\section{Cell culture}

For Western blot and immunofluorescence experiments, HeLa cells and the macrophage cell line RAW 264.7 (LGC Standards, USA) were used. Both cell lines were serum-starved overnight and RAW cells were interferon (IFN) $\gamma$-stimulated (100 U/ml, R\&D Systems, Germany) for 1 hour the next day. For co-culture and iron experiments, $1 \mathrm{mmol} / \mathrm{L}$ of soluble iron (ferric ammonium citrate) was added to the RAW 264.7 cells (to a final concentration of $100 \mu \mathrm{mol} / \mathrm{L}$ ) and allowed to permeate the membrane for 1 hour at $37^{\circ} \mathrm{C}$. Simultaneously, HeLa cells were CVB3-infected (MOI of 5) for 1 hour by adding the virus to the medium without further washes. After 1 hour, HeLa cells were added to the RAW 264.7 cells and investigated at specific time periods. As controls, iron-free and uninfected cells were used. For iNOS inhibition experiments, 


\section{Cellular Physiology and Biochemistry}

Cell Physiol Biochem 2014;33:52-66

DOI: $10.1159 / 000356649$

Published online: January 02, 2014

C) 2014 S. Karger AG, Basel

www.karger.com/cpb

RAW 264.7 cells were pre-treated for 1 hour with $1 \mathrm{mmol} / \mathrm{L} \mathrm{NG-nitro-L-arginine} \mathrm{methylester} \mathrm{(L-NAME}$ Sigma-Aldrich, Germany). For HO-1 inhibition experiments, RAW 264.7 macrophages were pre-treated with 20mmol/L tin mesoporphyrin (SnMP) for 6 hours (Cayman Chemicals, USA) before adding iron and/or CVB3-infected HeLa cells overnight. Cell lysates were blotted on PVDF membranes or used to extract RNA.

\section{Immunoblotting analysis}

Samples of frozen murine hearts or cell lysates were minced and homogenized in ice cold lysis buffer (Cell Signalling Technology, Danvers, MA, USA) supplemented with Complete EDTA-free Protease Inhibitor Cocktail (Roche, Mannheim, Germany) at recommended concentrations. $30 \mu \mathrm{g}$ of the extracted proteins were separated on a 10\% polyacrylamide gel and transferred to PVDF membranes. After blocking with 5\% non-fat dry milk in PBS for 1 hour at room temperature, blots were incubated overnight at $4{ }^{\circ} \mathrm{C}$ with rabbit anti-iNOS (Abcam, UK), rabbit anti-HO1 (Abcam, UK) or rabbit anti- $\beta$-tubulin (Cell Signalling Technology, Danvers, MA, USA). All primary antibodies were used at a 1:1000 dilution. Secondary peroxidase-conjugated goat anti-rabbit IgG antibodies (Santa Cruz Biotechnology, Santa Cruz, CA, USA, 1:2000 in PBS/5\% non-fat dry milk) were used for chemiluminescent protein detection with SuperSignal West Pico substrate (Pierce, USA). Target protein levels were quantified by ImageJ image analysis software (National Institutes of Health $(N I H)$, Bethesda, MD) and normalized to $\beta$-tubulin.

\section{Transfection with HO-1 siRNA}

HeLa cells $\left(2 \times 10^{4}\right)$ were grown in 96-wells plate (Nunc, Roskilde, Denmark) in serum and antibioticfree medium and transfected at 60-70\% confluency. After washing with Opti-MEM (Invitrogen, Karlsruhe, Germany) cells were overlaid with the transfection mixture containing PromoFectin-siRNA reagent $(5 \mu \mathrm{l}$, PromoCell, Heidelberg, Germany) and commercially available siRNA against hHO-1 (1 $\mu$ g, Santa Cruz Biotechnology, Santa Cruz, CA, USA) in a final volume of $200 \mu \mathrm{l}$ Opti-MEM. Six hours before assay, iron $(100 \mu \mathrm{mol} / \mathrm{L})$ and/or CVB3 (at MOI of 5) were added to the cells. siRNA containing a scrambled sequence was used as control. HO-1 siRNA transfection efficiency in the respective samples was determined by qRTPCR.

Immunofluorescence (IF)

Confluent CVB3-infected and/or iron treated HeLa cells or RAW 264.7 macrophages were fixed in 4\% paraformaldehyde solution in PBS (15 minutes, room temperature) and permeabilized by $0,1 \%$ Triton X-100 solution in PBS (20 minutes, room temperature). Cells were incubated with specific primary antibodies (rabbit HO-1, rabbit iNOS (Abcam, Cambridge, UK), rabbit caspase-3 (Cell Signalling Technology, Danvers, MA, USA) and Cox mAB 31A2 (CVB3 VP1) (Mediagnost, Reutlingen, Germany), diluted 1:100 for HO-1, iNOS and 1:400 for CVB3 VP1 and caspase- 3 overnight at $4^{\circ} \mathrm{C}$. Cells were further incubated with Alexa-488 antirabbit and Alexa-594 anti-mouse (Invitrogen, Karlsruhe, Germany), respectively. For negative controls, cells were treated omitting the first antibody. Images were taken with the help of a Zeiss Axiovert 2000 microscope equipped with an Axiocam HR camera and photos were acquired with a 40x Plan-Apochromat objective. For the analysis of caspase-3 signal intensity, the ImageJ analysis software (National Institutes of Health $(N I H)$, Bethesda, MD) was used.

\section{Nitric oxide production}

One way to investigate NO production in biological systems is to measure nitrite, which is the stable and non-volatile breakdown product of NO. For this, Griess reagent system was employed (Promega, Germany). Briefly, $50 \mu \mathrm{l}$ of culture supernatant was incubated with an equal volume of Griess reagent (0.5\% sulfanilamide, $0.05 \% \mathrm{~N}$-(1-naphthyl)-ethylenediamine dihydrochloride in $2.5 \% \mathrm{H}_{3} \mathrm{PO}_{4}$ ) in 96 -well tissue culture plate for 10 minutes at room temperature. The absorbance at $550 \mathrm{~nm}$ was measured with a microplate reader (iMark Microplate Absorbance Reader, Bio-Rad, Münich, Germany) including nitrite standards.

\section{Statistical analysis}

In statistical analyses data are presented as the mean \pm SEM. Data were analyzed by using GraphPad InStat3 software for Windows (San Diego, CA, USA) and the statistical significance was estimated by ANOVA with Tukey-Kramer multiple comparison post test. Values of $\mathrm{p}<0.05$ were considered to be statistically significant. 
Fig. 1. Increased iron deposition in susceptible SWR/J mice and its effect over viral replication in vitro. A. Susceptible SWR/J (a, c, e) but not resistant C57BL/6 (b, d, f) mice show iron deposits (c) within coxsackievirus-infected myocytes (e, CVB3 VP1 protein). B. Increased iron deposition in macrophages (arrows) and cardiomyocytes of susceptible CVB3-infected SWR/J mice in the course of infection. C. Quantification of increased enterovirus RNA (qRT-PCR) and infectious virions (plaque assay) after iron treatment in HeLa cells; results represent mean \pm SD of three independent experiments $\left({ }^{* *} \mathrm{p}<0.01, \quad * \mathrm{p}<0.05\right)$. Scale bar: c, d, e, f 100 $\mu \mathrm{m} ; 6 \mathrm{~d}$ pi $100 \mu \mathrm{m}, 9 \mathrm{~d}$ pi $200 \mu \mathrm{m}, 16 \mathrm{~d}$ pi, 2 months pi $30 \mu \mathrm{m}$.
A

A
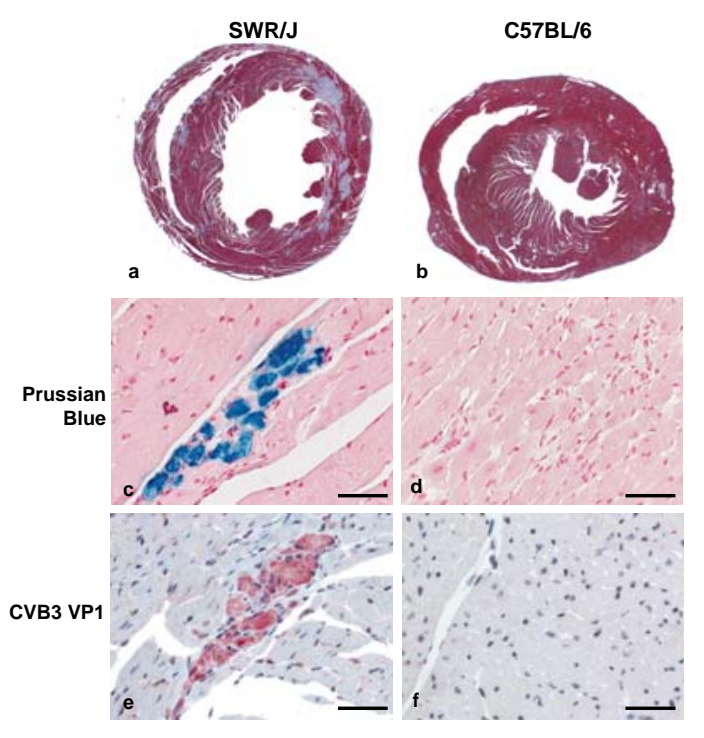

B

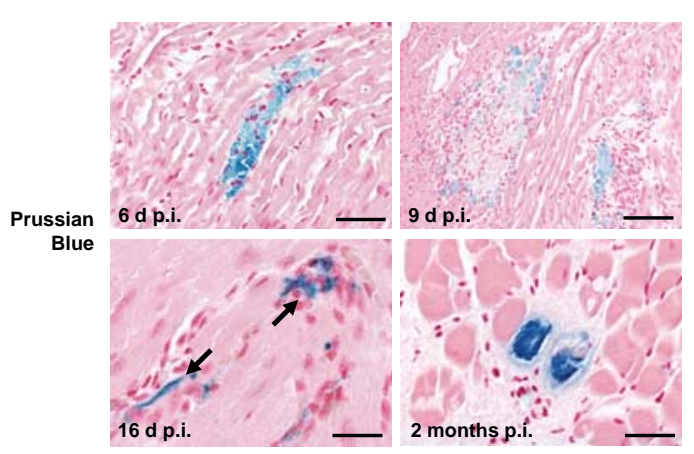

C

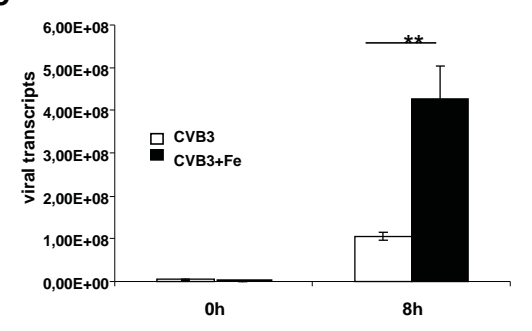

\section{Results}

Increased iron deposition in hearts of CVB3-infected SWR/J mice and the effect of iron on viral replication

We have previously shown that SWR/J mice but not C57BL/6 mice are susceptible for the development of chronic CVB3 myocarditis which is associated with severe remodelling processes at later stages of infection [14]. Interestingly, in this study we found that in the infected and damaged myocardium of SWR/J mice (Fig. 1A, Trichrome) the iron deposits are present within infected myocytes, as shown by Prussian blue (c) and immunohistochemical CVB3 viral protein-1 (VP1) stainings (e) in corresponding tissue sections. In contrast, CVB3infected C57BL/6 mice, which are resistant to chronic myocarditis, did not reveal significant cardiac damage (b), iron deposits (d) or persistent virus infection (f). We also found that in the course of chronic myocarditis in SWR/J mice, iron is not only incorporated in damaged myocytes but also in macrophages in significant amounts starting at 16 days p.i. (Fig. 1B). In myocytes, iron accumulation was detectable already from day 6 p.i. on. Interestingly, in the 


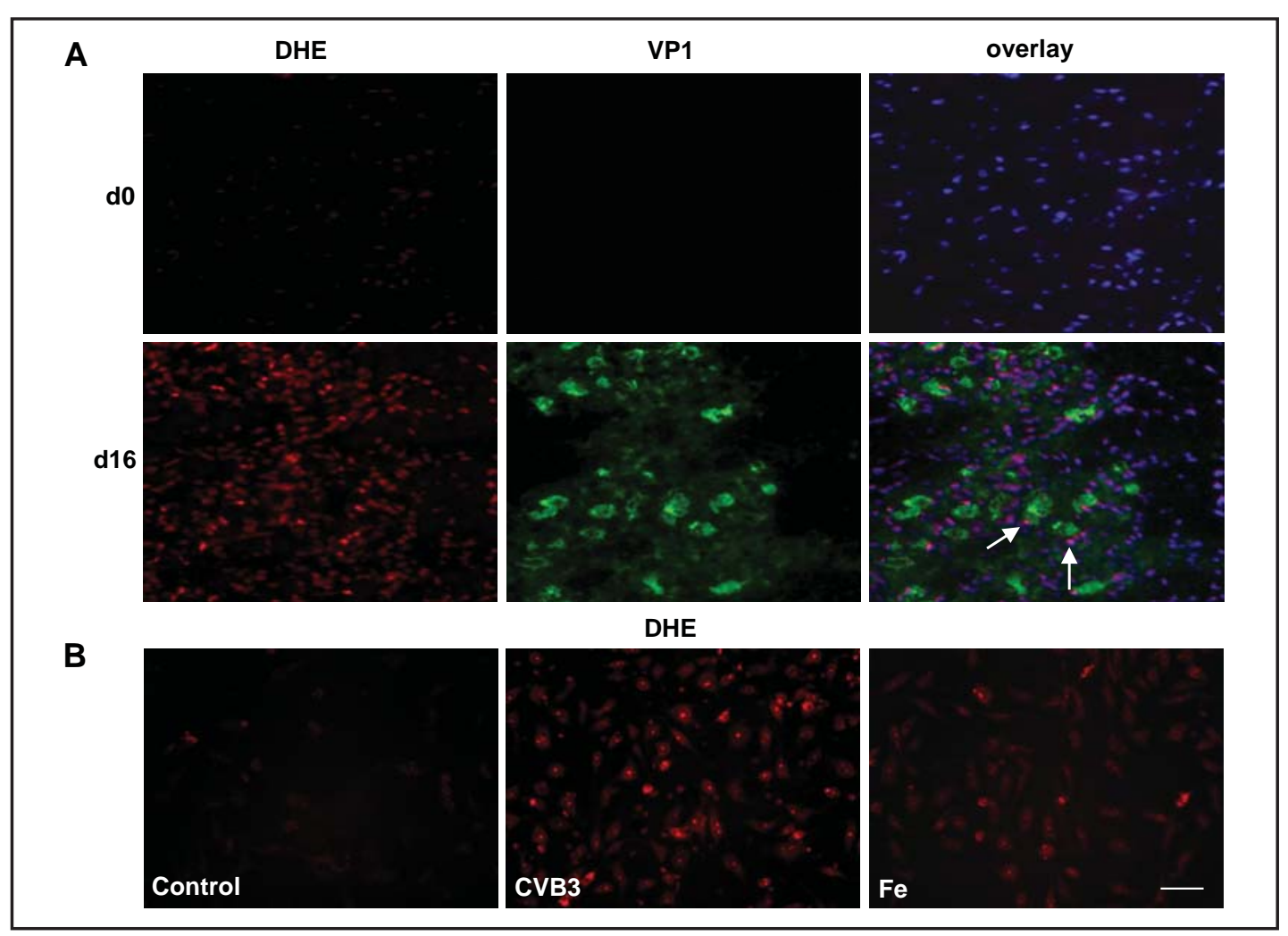

Fig. 2. Oxidative stress in iron-loaded CVB3-infected hearts of SWR/J mice. A. Superoxide which is released from infiltrating macrophages (white arrows) and detected by staining with the superoxide-sensitive dye dihydroethidium DHE (red fluorescence), shows increased signal intensity in close vicinity of infected myocytes (16 days p.i; co-staining with CVB3 VP1 (green) and DAPI). B. Oxidative stress (DHE staining) in CVB3 and iron treated RAW 264.7 macrophages. Scale bar: $200 \mu \mathrm{m}$.

course of myocarditis the majority of iron deposits were observed in myocytes located at the border to uninfected tissue ( 9 days p.i). Even after the decline of inflammation numerous damaged cardiomyocytes were found to be heavily loaded with iron, as exemplarily shown in a heart 2 months p.i.. To gain more insight into the interaction of iron and viral replication, we quantified the enteroviral RNA by qRT-PCR in untreated and iron-treated $(100 \mu \mathrm{mol} / \mathrm{L})$ CVB3-infected HeLa cells, a well defined cell culture model of CVB3 myocarditis. Irontreated samples revealed a 4-fold increase in the number of viral RNA transcripts compared to untreated infected cells (Fig. 1C). Correspondent to the results in qRT-PCR, we found in plaque assay experiments a significant increase of infectious virions in HeLa cells in presence of iron (Fig. 1C).

\section{Oxidative stress is induced by CVB3 replication and iron}

In order to gain more insight into the effects of iron in the virus-infected myocardium, we investigated the stress conditions in these hearts. CVB3 myocarditis was found to be associated with increased oxidative stress mainly within infected areas, as visualized by colocalization of CVB3 VP1 in myocytes, with infiltrating macrophages as source of superoxide (Fig. 2A, white arrows, 16 days pi.). In contrast, resistant C57BL/6 mice revealed less oxidative stress which correlates with low immune cell activity after CV3B infection (data not shown). Further information is provided in iron-loaded and virus-stimulated RAW 264.7 macrophages, revealing an upregulation of superoxide production under both conditions. As expected, replicating CVB3 was found to stress the cells to a higher extent than iron (Fig. 2B). Cultivated untreated macrophages were found to be negative for superoxide expression. 


\section{Cellular Physiology and Biochemistry}

Cell Physiol Biochem 2014;33:52-66

\begin{tabular}{l|l}
\hline DOI: $10.1159 / 000356649$ & (C) 2014 S. Karger AG, Basel
\end{tabular}

www.karger.com/cpb

Ursu et al.: Heme Oxygenase-1 in Enteroviral Myocarditis

Fig. 3. iNOS mRNA and protein detection in CVB3-infected hearts and cultivated macrophages. A. Strong iNOS mRNA expression by infiltrating macrophages in the heart of acutely CVB3-infected SWR/J mice shown by radioactive ISH (8 days p.i., scale bar: $100 \mu \mathrm{m}$ ). B. Representative immunoblot for iNOS protein expression in SWR/J heart extracts revealing highest levels during acute myocarditis (8 days p.i.). C. iNOS protein expression in iron or CVB3-treated RAW 264.7 macrophages 8 hours p.i., as shown by IF. Scale bar: $200 \mu \mathrm{m}$.

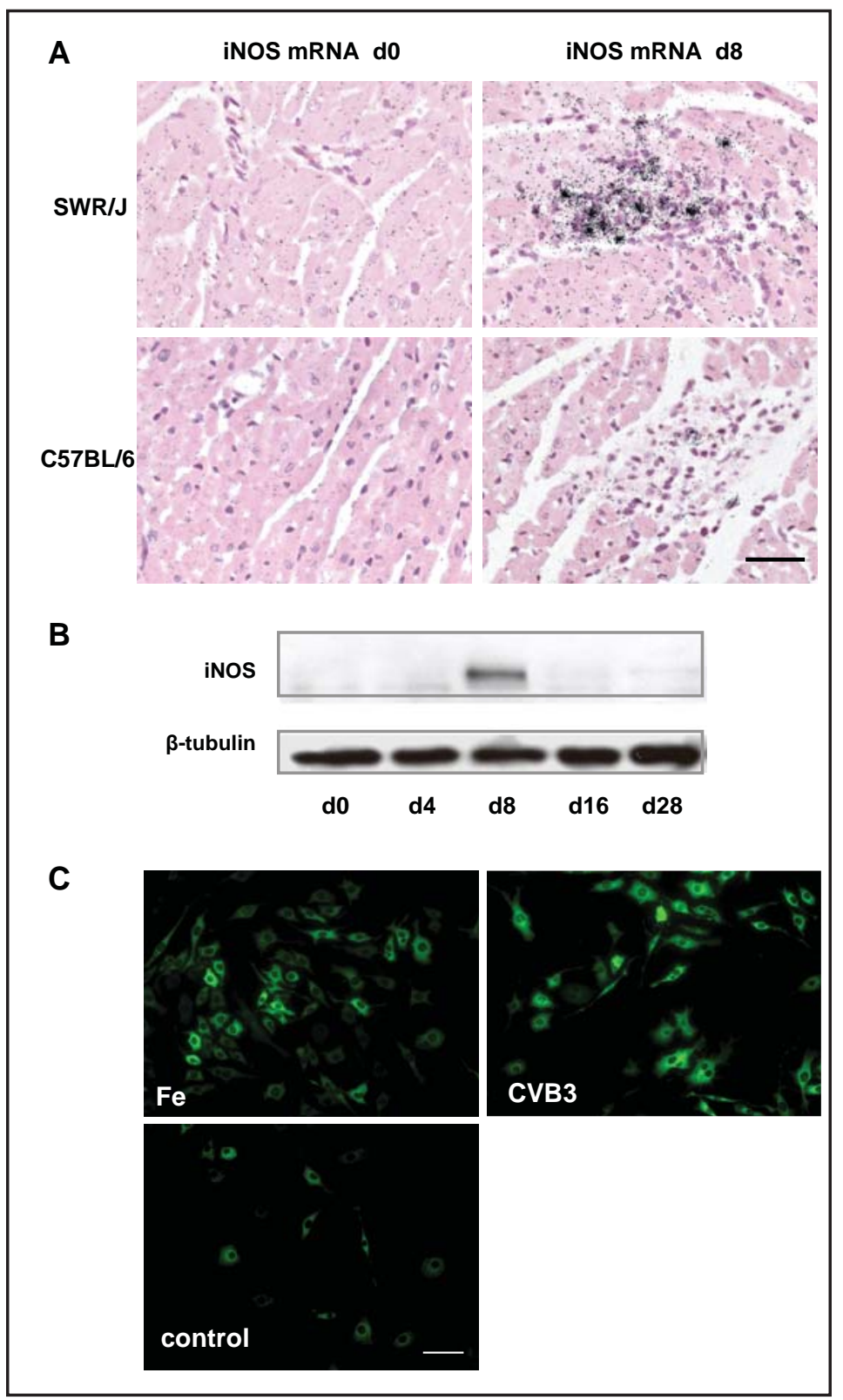

Induction of iNOS by iron and CVB3

Upregulation of the iNOS is known to be a major cause of oxidative stress in the heart. We have previously shown that iNOS expression in cardiac macrophages contributes to the immunopathology of ongoing myocarditis in susceptible ABY/SnJ mice [5]. In order to evaluate whether iron influences iNOS expression in the course of CVB3 myocarditis, hearts of iron-loaded SWR/J mice were analyzed in the course of myocarditis. In contrast to resistant C57BL/6 mice, SWR/J mice reveal numerous iNOS mRNA positive cells in areas of severe inflammation following CVB3 infection (Fig. 3A). Correspondent to the in situ hybridization results, the maximum of iNOS protein expression was observed 8 days pi. (Fig. 3B). In order to evaluate the effect of iron and CVB3 infection on iNOS expression in vitro, cultivated RAW 264.7 macrophages were investigated by immunofluorescence stainings. Corresponding to our observations of superoxide production, we found an upregulation of iNOS protein levels in both, CVB3-stimulated as well as in iron treated RAW 264.7 macrophages, when compared to untreated control cells (Fig. 3C). 


\section{Cellular Physiology and Biochemistry}

Cell Physiol Biochem 2014;33:52-66

Fig. 4. HO-1 mRNA and protein detection in CVB3-infected hearts and cultivated cells. A. Detection of HO-1 mRNA by in situ hybridization (a, b, d and e) and protein by immunohistochemistry (c and f) within macrophages (arrows) and cardiomyocytes (arrowhead) in hearts of infected susceptible and resistant mice, revealing high HO-1 mRNA and protein expression in susceptible SWR/J mice during acute CVB3 myocarditis (8 days p.i.). Scale bar: $100 \mu \mathrm{m}$ (a-f). B. HO-1 IF in CVB3-infected and/or iron-treated HeLa cells show increased protein expression 8 hours p.i in the nucleus (CVB3) or cytoplasm (iron) or both (CVB3/iron). Scale bar: $20 \mu \mathrm{m}$. C. HO-1 representative immunoblot and densitometric analyses of three independent experiments reveal increased HO-1 protein expression in RAW 264.7-HeLa cell cocultures 8 hours p.i. in presence of iron, showing that CVB3 and iron potentiate each others activity (significance vs. untreated cocultured control cells: ${ }^{*} \mathrm{p}<0.05,{ }^{* *} \mathrm{p}<0.01$ and $* * * \mathrm{p}<0.001$ ).

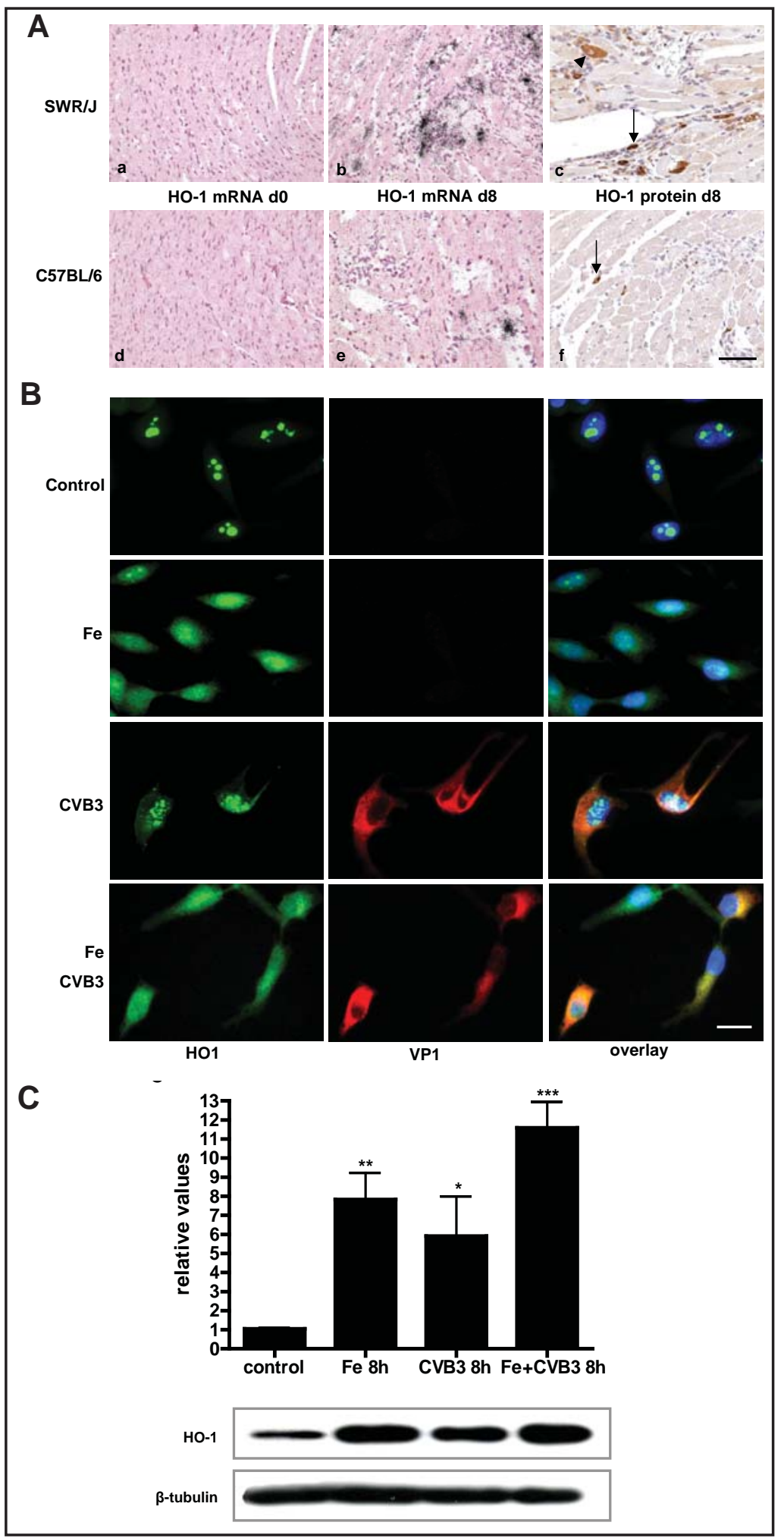

Heme-oxygenase 1 is upregulated by iron and CVB3

Nitric oxide is known as one of the most potent inducers of HO-1 gene expression in various tissues in vivo [15] as well as in different cell types in vitro [16]. In order to evaluate whether the oxidative stress reactions may influence HO-1 expression in vivo, we first investigated hearts of CVB3-infected SWR/J and C57BL/6 mice. Susceptible SWR/J mice 

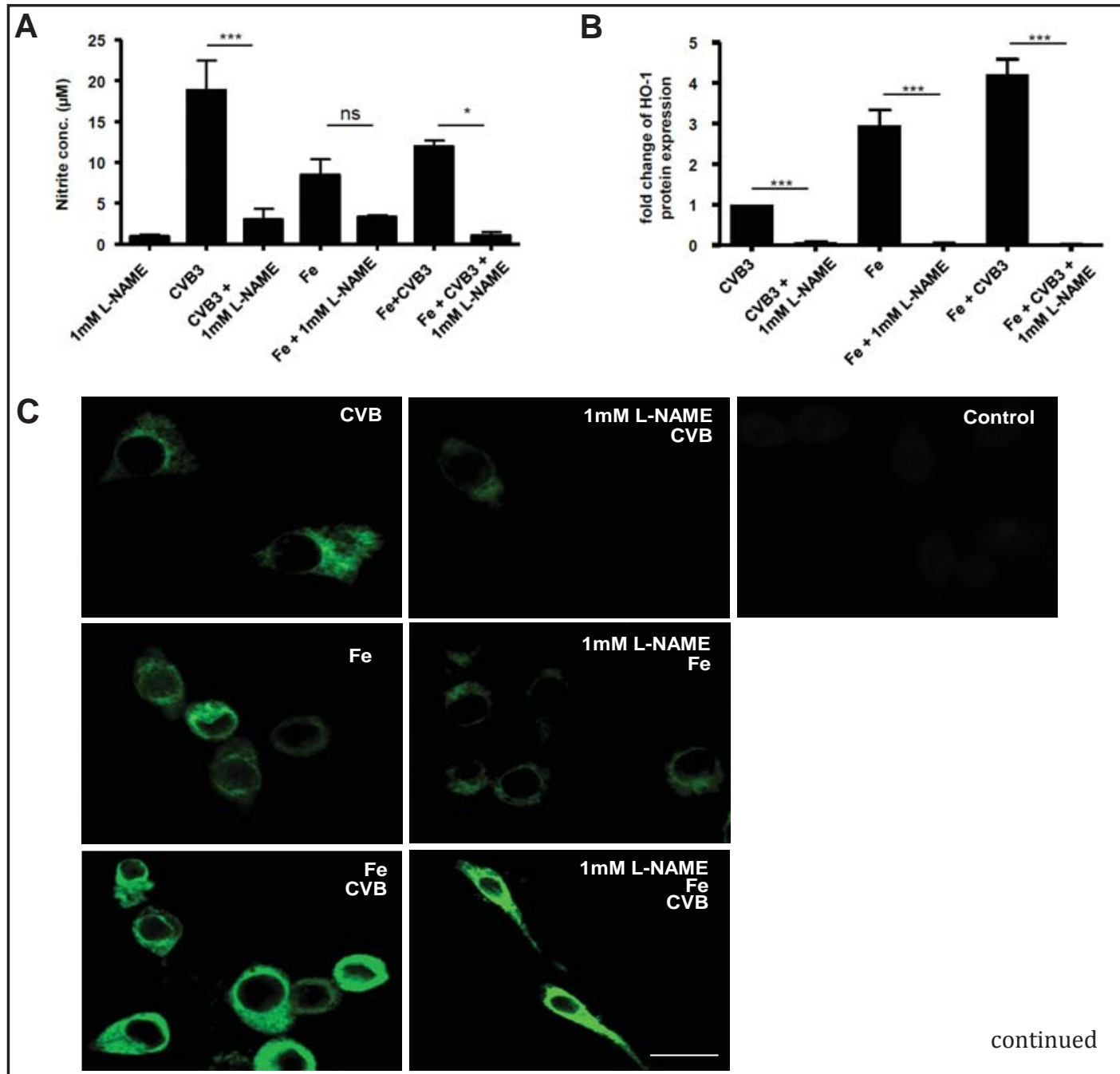

continued

reveal comparably higher amounts of HO-1 mRNA and protein expressing immune cells in cardiac inflammatory lesions compared to C57BL/6 mice at any time of infection (data not shown), reaching a maximum during the acute phase of the disease (8 days p.i., Fig. 4A).

A detailed immunohistochemical study at the cellular level revealed that increased HO-1 protein expression is mainly observed in macrophages (arrows) but is also detected in single cardiomyocytes (arrowhead).

In order to investigate the effects of iron and CVB3 on HO-1 expression in vitro, HeLa cells were treated by either iron and/or CVB3. As demonstrated in Figure 4B, immunofluorescence of untreated control cells illustrates the presence of HO-1 in nucleoli, a finding which was previously reported by Li Volti in cultured astrocytes [17]. Incubation of cells with iron and/ or CVB3 resulted in the upregulation of HO-1 protein in different compartments of the cells and revealed a spread of $\mathrm{HO}-1$ within the nucleus (CVB3) as well as over the cytoplasm (iron) or both (CVB3/iron). To simulate the interaction of myocytes and macrophages observed in vivo with regard to HO-1 expression, we performed western blot analyses of cytoplasmic protein extracts obtained from RAW 264.7 -HeLa cell cocultures (Fig. 4C). Compared to untreated cells, a significant potentiation in H0-1 protein expression was observed after addition of CVB3 ( $<<0.05)$, iron $(\mathrm{p}<0.01)$ or iron/CVB3 $(\mathrm{p}<0.001)$.

$\mathrm{HO}-1$ expression is influenced by iNOS activity and mediates oxidative stress

As mentioned before, sustained iNOS activity has been proven to result in ongoing cardiac injury in CVB3 myocarditis. As it is well known that changes in iNOS protein strongly 


\section{Cellular Physiology and Biochemistry}

Cell Physiol Biochem 2014;33:52-66

\begin{tabular}{l|l}
\hline DOI: $10.1159 / 000356649$ & (C) 2014 S. Karger AG, Basel
\end{tabular}

Fig. 5. HO-1 is modulated by NO production and increases oxidative stress in RAW-264.7 macrophages/ HeLa cocultures. A. Nitrite production is decreased by L-NAME in macrophages which were treated with CVB3 $\left.{ }^{* * *} \mathrm{p}<0.001\right)$, iron (p> 0.05), or iron /CVB3 $\left({ }^{*} \mathrm{p}<0.01\right)$. B. Decreased HO-1 protein expression when NO production is inhibited by L-NAME in immunoblots (densitometric analyses of three independent experiments $\left.{ }^{* * *} \mathrm{p}<0.001\right)$. C. Immunofluorescence shows decreased HO-1 expression in CVB3, iron and CVB3/irontreated cocultured cells in presence of L-NAME. Scale bar: $20 \mu \mathrm{m}$. D. Superoxide production, as detected by DHE staining (red) in RAW 264.7 macrophages (green) which were cocultured with CVB3-infected HeLa cells, is decreased in presence of HO-1-downregulating tin mesoporphyrin (SnMP, 20mM). Before the experiment, RAW 264.7 macrophages were loaded with CellTracker green in order to differentiate them from HeLa cells. Scale bar: $50 \mu \mathrm{m}$.

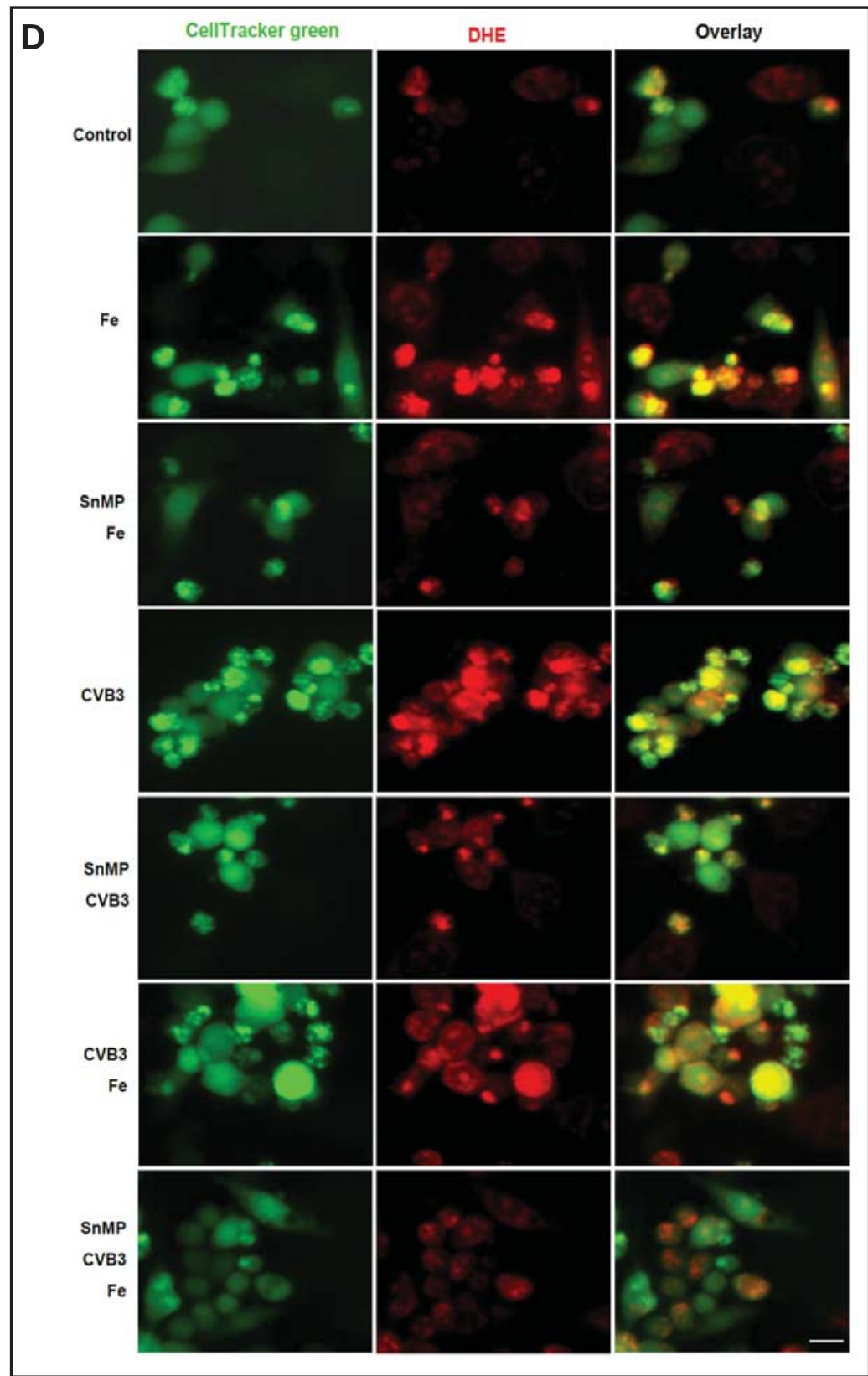

correlate with changes in nitrites, we measured nitrite levels in RAW 264.7/HeLa cell cocultures and found, as expected, an upregulation of nitrite levels upon iron or/and CVB3 infection. Incubation with L-NAME resulted in significantly decreased levels of nitrite in CVB3 and CVB3/iron treated cells (Fig. 5A). In order to investigate whether HO-1 is regulated via iNOS, we blocked iNOS activity in L-NAME treated RAW 264.7 -HeLa cell cocultures. Western blot analysis revealed that inhibition of iNOS activity by L-NAME significantly reduces HO-1 protein expression in CVB3, iron and CVB3/iron treated cells $((\mathrm{p}<0.001)$ Fig. $5 B)$. This observation is supported by immunofluorescence proving a reduction in the HO-1 signal intensity upon blocking of iNOS by L-NAME followed by iron or/and CVB3 treatment (Fig. 5C), indicating that iNOS modulates HO-1 expression.

Superoxide production contributes to oxidative stress and can be observed in cells upon CVB3 infection and treatment with iron. In order to evaluate the contribution of HO-1 expression on the generation of superoxide, HO-1 was blocked by SnMP. As shown in Figure 5D, we found a clear reduction in superoxide production after HO-1 inhibition upon iron or/ 


\section{Cellular Physiology and Biochemistry}

Cell Physiol Biochem 2014;33:52-66

DOI: $10.1159 / 000356649$

Published online: January 02, 2014

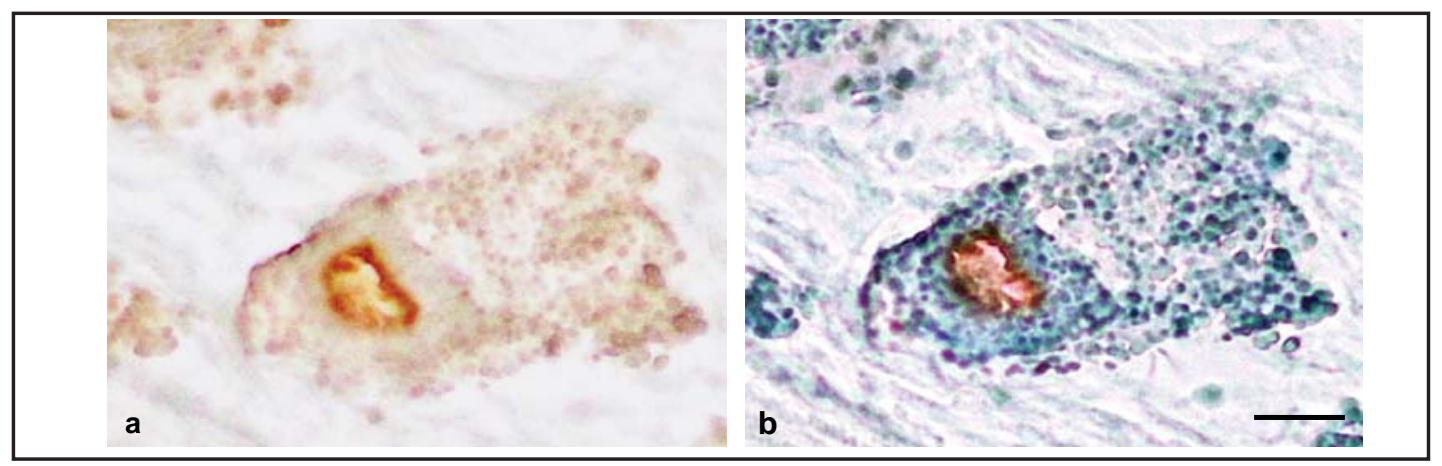

Fig. 6. Apoptosis and iron in cardiomyocytes 2 months p.i. of CVB3-infected SWR/J mice. Immunohistochemistry for activated caspase-3 (a) in a cardiomyocyte 2 months p.i. CVB3 followed by Prussian blue stain (b) shows that iron-loaded cardiomyocytes are undergoing apoptosis. Scale bar: $10 \mu \mathrm{m}$.

and CVB3 treatment demonstrating that HO-1 expression accounts for oxidative activities in CVB3 infection.

\section{HO-1 expression and apoptosis}

In order to investigate the contribution of CVB3 persistence, increased iron storage as well as of oxidative stress on the survival of myocytes in vivo, we investigated CVB3-infected hearts of SWR/J mice by light microscopy. As exemplarily demonstrated in Figure 6, we observed activated caspase-3 expression (a) in those cardiomyocytes which are also loaded with iron (b) at later stages of the disease. These findings indicate that iron containing myocytes undergo apoptosis in persistently CVB3-infected mice, which corresponds well to our observations in human inflammatory iron-induced cardiomyopathy (data not shown).

To further determine whether the host cell death machinery is activated by iron and CVB3 infection in vitro, HeLa cells were used as a model system. As demonstrated in Figure 7A, caspase- 3 which was found to be activated by iron, localizes mainly around the nucleus. Also, CVB3 induced caspase-3 activation, however with a primary localization in the cytoplasm. The presence of both stimuli, CVB3 and iron, induces nuclear condensation in addition to activation of caspase-3.

To delineate the role of HO-1 in caspase- 3 activation of iron- and CVB3-treated cells, we used an HO-1 siRNA approach. In parallel to the experiments presented in Figure 7A, we performed immunofluorescence staining for the detection of activated caspase-3, however in presence of HO-1 siRNA. HO-1 silencing resulted in a significant reduction of signal intensity of caspase-3 signals in iron and/or CVB3 treated cells (Fig. 7B) compared to the correspondent results in Fig. 7A. Image analysis of caspase-3 signal intensity in CVB3-infected and irontreated samples reveal a significant downregulation of caspase-3 expression in presence of HO-1 siRNA (Fig. 7C) $(\mathrm{p}<0.001)$. These results substantiate the role of HO-1 in caspase-3 activation which is also observed in vivo in iron-loaded myocytes of CVB3-infected mice (compare Fig. 6). In order to verify the efficiency of the HO-1 siRNA approach, we previously tested by qRT-PCR the amount of HO-1 mRNA in relation to correspondent hHPRT mRNA levels in HeLa cells which were transfected with anti-human HO-1 siRNA in CVB3 and irontreated cells. HO-1 mRNA was found to be decreased to more than 50\% after HO-1 gene silencing in CVB3- and iron-treated cells (Fig. 7D) ( $<<0.05)$.

\section{Discussion}

CVB3 infection triggers macrophages to infiltrate the heart, releasing iNOS [5] and superoxide anions which are suggested to contribute to oxidative stress and cardiac damage in chronic viral myocarditis [7]. Here, we demonstrate that in the chronic course of the disease macrophages of SWR/J mice, but not those of resistant C57BL/6 mice, reveal high amounts of iron, which is also known to upregulate ROS production [18]. Recently, a strong 


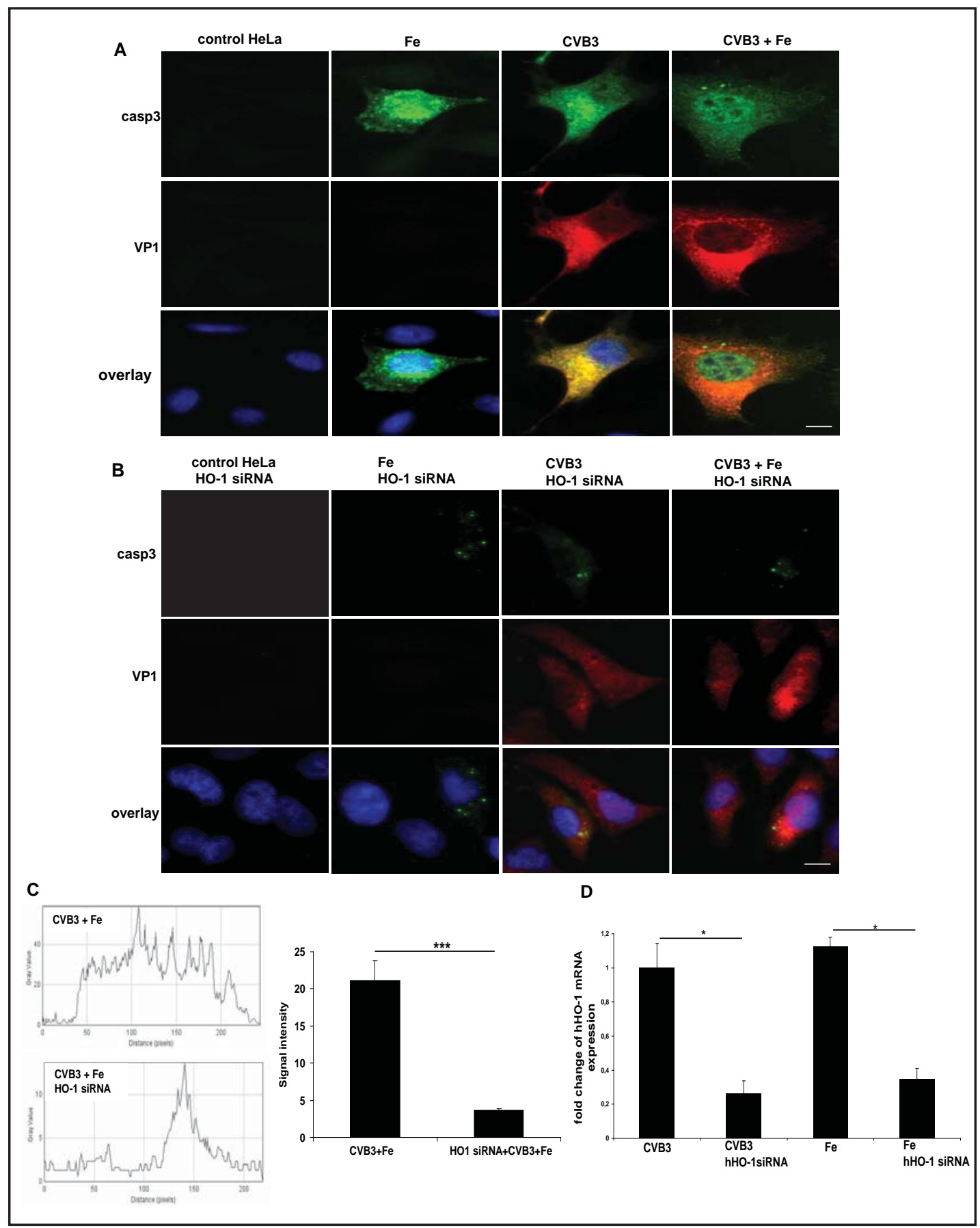

Fig. 7. HO-1 silencing in HeLa cells correlates with reduction of caspase-3 activation. A. Activated caspase-3 protein expression (green) in HeLa cells after iron and/or CVB3 treatment. CVB3 VP1 protein (red) as well as caspase-3 (green) are evenly distributed in the cytoplasm of the cell. The simultaneous presence of CVB3 and iron induces activation of caspase-3 in the nucleus and cytoplasm. B. Decreased caspase-3 protein expression in cells is observed by downregulation of HO-1 using siRNA in all treated samples. C. Representative quantification of caspase-3 expression with and without HO-1 siRNA in CVB3-infected and iron-treated cells. Pixel intensities which were determined for individual cells of similar sizes along a line crossing the diameter of the cell outside of the nucleus reveal a significant downregulation of caspase- 3 in presence of HO-1 siRNA $\left({ }^{* * *} \mathrm{p}<0.001\right)$. D. The efficiency of the HO-1 siRNA approach was first tested by qRT-PCR. HO-1 mRNA was found to be decreased to more than 50\% after HO-1 gene silencing in CVB3 and iron-treated cells (Fig. 7C) $\left({ }^{*} \mathrm{p}<0.05\right)$. Scale bar: $10 \mu \mathrm{m}$. 


\section{Cellular Physiology and Biochemistry}

Cell Physiol Biochem 2014;33:52-66

DOI: 10.1159/000356649

online: January 2, 2014

quantitative trait locus identified on mouse chromosome 9 was proven to differentially affect the macrophage iron burden in SWR/J and C57BL strains [19], concluding that genetic background might influence the intracellular iron metabolism in the two mouse strains. In our manuscript, we provide new evidence that differences in macrophage iron content also influences the outcome of the enteroviral myocarditis in these genetically different mouse strains, substantiating the relevance of the host' genetics in the pathogenesis of the disease. Interestingly, incorporation of iron was not only observed in macrophages, but also in CVB3-infected cardiomyocytes of SWR/J mice within areas of increased oxidative activities. Previously it was shown in rat cardiomyocytes that iron induces enhanced generation of ROS and stimulates iNOS activity, thus contributing to heart damage [20]. The enhanced cardiac oxidative activities seen in CVB3-infected susceptible mice were correlated with the upregulation of the HO-1 mRNA and protein in macrophages, as well as in infected cardiomyocytes. One of the most potent inducers of HO-1 gene expression for various tissues in vivo [15], as well as for different cell types in vitro [16] is NO. Increased NO production was described to contribute to immunopathology in ongoing murine CVB3 myocarditis [5]. On the other hand it was published that in presence of excessive NO, produced by either iNOS DNA overexpression or treatment with excessive concentrations of an NO donor, CVB3 replication is inhibited in vitro- however only during the initial stages of infection when NO was added before or latest 1 hour post infection [21]. Previously it was shown that in rat liver iron overload triggers oxidative stress at early times, with upregulation of iNOS expression involving the ERK/NF-kappaB pathway at later times [22], and that in rat hearts induction of cardiac iNOS promotes myocardial injury due to oxidative stress [23]. Our observations in susceptible SWR/J mice revealing chronic myocarditis indicate that the NO/HO-1 pathway is likely induced by both, CVB3 as well as iron. In vitro experiments revealed that blockage of NO production by L-NAME decreases HO-1 protein expression in CVB3 and iron-treated cells, underlining the role of $\mathrm{NO}$ in modulating $\mathrm{HO}-1$ activity. Although a low expression level of HO- 1 is described to be associated with protection against oxidative stress [24], an excess expression of this enzyme was found to be associated with increased oxygen cytotoxicity, being detrimental for the cell physiology [25]. Notably, in our cell culture experiments we observed not only a cytoplasmic, but also an intranuclear localization of HO-1 under iron and CVB3 treatment, which might be explained by the attempt of the cell to provide cytoprotection against oxidative stress by regulating the expression of antioxidant genes [26].

Both superoxide and iron have been described to contribute to cardiac damage and cardiomyocyte apoptosis $[27,28]$. We observed an activation of caspase-3 in vitro as well as in vivo in cardiomyocytes in association with iron overload and CVB3 infection. Morevover, we found that these apoptotic processes could be inhibited in HO-1 siRNA experiments. In previous experiments with leukemia cells, it was also demonstrated that HO-1 expression contributes to apoptosis mediated by oxidative stress [29]. However, in a model of cardiac ischemia and reperfusion, $\mathrm{HO}-1$ has been described to attenuate cardiomyocytic apoptosis [30] by reducing NF- $\kappa B$ and AP-1 nuclear translocation, thereby reducing the level of caspase-3 activation and the surge of inflammatory cytokines [31]. On the other hand, more recent findings showed additional deleterious effects of $\mathrm{HO}-1$ by proving that systemic $\mathrm{HO}-1$ overexpression aggravates pressure overload-induced cardiac hypertrophy [32].Importantly, increased cellular iron deposition not only contributes to the heart disease by potentiating oxidative stress and apoptosis, but also by increasing viral replication with a 4 -fold increase in CVB3 transcript numbers in iron-treated HeLa cells. So far, the molecular basis by which iron alters CVB3 replication is unknown. However, also in other virus infections, such as HIV-1, an increased iron load in macrophages was found to be associated with increased viral replication [33]. Similarly, hepatitis B virus mRNA and DNA expression was shown to be increased by iron. Interestingly, miRNA expression studies revealed modulatory effects of iron on HBV replication [34]. Whether differential miRNA expression might also play a role for the increased CVB3 replication in our susceptible animals has to be investigated in further experiments. 


\section{Cellular Physiology and Biochemistry}

Cell Physiol Biochem 2014;33:52-66

\begin{tabular}{l|l}
\hline DOI: $10.1159 / 000356649$ & (c) 2014 S. Karger AG, Basel
\end{tabular}

\begin{tabular}{l|l} 
Published online: January 02, 2014 & www.karger.com/cpb
\end{tabular}

Ursu et al.: Heme Oxygenase-1 in Enteroviral Myocarditis

In summary, we have demonstrated that HO-1 mediates a pathologic crosstalk between macrophages and cardiomyocytes in CVB3-induced myocarditis, thus resulting in increased oxidative stress and the evolvement of apoptotic cardiomyocytes, finally contributing to heart failure. From these data it can be concluded that in genetically susceptible mice iron substantially increases the detrimental effects of CVB3 by the NO/HO-1 pathway, thus increasing the pathogenicity and injury in the heart. As a consequence, patients suffering from chronic heart failure should not be prescribed iron unless it is proven that this severe disease is not the result of a chronic enterovirus infection.

\section{Conflict of Interest}

None.

\section{Acknowledgements}

We thank Sandra Bundschuh for excellent technical assistance. This work was supported by a grant of the BMBF (No. 01EZ0817) and the Deutsche Forschungsgemeinschaft (SFBTR19) to K.K. and R.K.

\section{References}

1 Martino TA, Liu P, Sole MJ: Viral infection and the pathogenesis of dilated cardiomyopathy. Circ Res 1994;74:182-188.

2 Haas GJ: Etiology, evaluation, and management of acute myocarditis. Cardiol Rev 2001;9:88-95.

-3 McManus BM, Chow LH, Wilson JE, Anderson DR, Gulizia JM, Gauntt CJ, Klingel KE, Beisel KW, Kandolf R: Direct myocardial injury by enterovirus: a central role in the evolution of murine myocarditis. Clin Immunol Immunopathol 1993;68:159-169.

4 Li K, Xu W, Guo Q, Jiang Z, Wang P, Yue Y, Xiong S: Differential macrophage polarization in male and female BALB/c mice infected with coxsackievirus B3 defines susceptibility to viral myocarditis. Circ Res 2009;105:353-364.

-5 Szalay G, Sauter M, Hald J, Weinzierl A, Kandolf R, Klingel K: Sustained nitric oxide synthesis contributes to immunopathology in ongoing myocarditis attributable to Interleukin-10 disorders. Am J Pathol 2006;169:2085-2093.

-6 Khadour FH, Panas D, Ferdinandy P, Schulze C, Csont T, Lalu MM, Wildhirt SM, Schulz R: Enhanced NO and superoxide generation in dysfunctional hearts from endotoxemic rats. Am J Physiol Heart Circ Physiol 2002;283:H1108-H1115.

7 Hiraoka Y, Kishimoto C, Takada H, Kurokawa M, Ochiai H, Shiraki K, Sasayama S: Role of oxygen derived free radicals in the pathogenesis of coxsackievirus B3 myocarditis in mice. Cardiovasc Res 1993;27:957961.

-8 Ryter SW, Otterbein LE, Morse D, Choi AM: Heme oxygenase/carbon monoxide signaling pathways: regulation and functional significance. Mol Cell Biochem 2002;234-235:249-263.

$\checkmark 9$ Vile GF, Basu-Modak S, Waltner C, Tyrrell RM: Heme oxygenase 1 mediates an adaptive response to oxidative stress in human skin fibroblasts. Proc Natl Acad Sci USA 1994;91:2607-2610.

-10 Anning PB, Chen Y, Lamb NJ, Mumby S, Quinlan GJ, Evans TW, Gutteridge JMC: Iron overload upregulates haem oxygenase 1 in the lung more rapidly than in other tissues. FEBS Lett 1999;447:111-114.

-11 Jankowska EA, Rozentryt P, Witkowska A, Nowak J, Hartmann O, Ponikowska B, Borodulin-Nadzieja L, Banasiak W, Polonski L, Filippatos G, McMurray JJV, Anker SD, Ponikowski P: Iron deficiency: an ominous sign in patients with systolic chronic heart failure. Eur Heart J 2010;31:1872-1880.

-12 Schwartz KA, Li Z, Schwartz DE, Cooper TG, Braselton WE: Earliest cardiac toxicity induced by iron overload selectively inhibits electrical conduction. J Appl Physiol 2002;93:746-751.

13 Li J, Lothar Schwimmbeck P, Tschope C, Leschka S, Husmann L, Rutschow S, Reichenbach F, Noutsias M, Kobalz U, Poller W, Spillmann F, Zeichhardt H, Schultheiss HP, Pauschinger M: Collagen degradation in a murine myocarditis model: relevance of matrix metalloproteinase in association with inflammatory induction. Cardiovasc Res 2002;56:235-247. 


\section{Cellular Physiology and Biochemistry}

Cell Physiol Biochem 2014;33:52-66

\begin{tabular}{l|l}
\hline DOI: $10.1159 / 000356649$ & (C) 2014 S. Karger AG, Basel
\end{tabular}

Ursu et al.: Heme Oxygenase-1 in Enteroviral Myocarditis

14 Klingel K, Hohenadl C, Canu A, Albrecht M, Seemann M, Mall G, Kandolf R: Ongoing enterovirus-induced myocarditis is associated with persistent heart muscle infection: quantitative analysis of virus replication, tissue damage, and inflammation. Proc Natl Acad Sci USA 1992;89:314-318.

-15 Willis D, Tomlinson A, Frederick R, Paulclark MJ, Willoughby DA: Modulation of heme oxygenase activity in rat brain and spleen by inhibitors and donors of nitric oxide. Biochem Biophys Res Commun 1995;214:1152-1156.

16 Datta PK, Lianos EA: Nitric oxide induces heme oxygenase-1 gene expression in mesangial cells. Kidney Int 1999;55:1734-1739.

-17 Li VG, Ientile R, Abraham NG, Vanella A, Cannavo G, Mazza F, Curro M, Raciti G, Avola R, Campisi A: Immunocytochemical localization and expression of heme oxygenase-1 in primary astroglial cell cultures during differentiation: effect of glutamate. Biochem Biophys Res Commun 2004;315:517-524.

18 Ryan TP, Aust SD: The role of iron in oxygen-mediated toxicities. Crit Rev Toxicol 1992;22:119-141.

19 Wang F, Paradkar PN, Custodio AO, Vey Ward D, Fleming MD, Campagna D, Roberts KA, Boyartchuk V, Dietrich WF, Kaplan J, Andrews NC: Genetic variation in Mon1a affects protein trafficking and modifies macrophage iron loading in mice. Nat Genet 2007;39:1025-1032.

-20 Munoz JP, Chiong M, García L, Troncoso R, Toro B, Pedrozo Z, Diaz-Elizondo J, Salas D, Parra V, Núñez MT, Hidalgo C, Lavandero S: Iron induces protection and necrosis in cultured cardiomyocytes: role of reactive oxygen species and nitric oxide. Free Radic Biol Med 2010;48:526-534.

-21 Zaragoza C, Ocampo CJ, Saura M, McMillan A, Lowenstein CJ: Nitric oxide inhibition of coxsackievirus replication in vitro. J Clin Invest 1997;100:1760-1767.

-22 Cornejo P, Varela P, Videla LA, Fernández V: Chronic iron overload enhances inducible nitric oxide synthase expression in rat liver. Nitric Oxide 2005;13:54-61.

23 Igarashi J, Nishida M, Hoshida S, Yamashita N, Kosaka H, Hori M, Kuzuya T, Tada M: Inducible nitric oxide synthase augments injury elicited by oxidative stress in rat cardiac myocytes. Am J Physiol Cell Physiol 1998;274:C245-C252.

-24 Dennery PA, Wong HE, Sridhar KJ, Rodgers PA, Sim JE, Spitz DR: Differences in basal and hyperoxiaassociated HO expression in oxidant-resistant hamster fibroblasts. Am J Physiol Lung Cell Mol Physiol 1996;271:L672-L679.

-25 Suttner DM, Dennery PA: Reversal of HO-1 related cytoprotection with increased expression is due to reactive iron. FASEB J 1999;13:1800-1809.

-26 Lin Q, Weis S, Yang G, Weng YH, Helston R, Rish K, Smith A, Bordner J, Polte T, Gaunitz F, Dennery PA: Heme oxygenase-1 protein localizes to the nucleus and activates transcription factors important in oxidative stress. J Biol Chem 2007;282:20621-20633.

-27 Li PF, Dietz R, von Harsdorf R: Superoxide induces apoptosis in cardiomyocytes, but proliferation and expression of transforming growth factor- $\beta 1$ in cardiac fibroblasts. FEBS Lett 1999;448:206-210.

28 Wang Y, Wu M, Al-Rousan R, Liu H, Fannin J, Paturi S, Arvapalli RK, Katta A, Kakarla SK, Rice KM, Triest WE, Blough ER: Iron-induced cardiac damage: role of apoptosis and deferasirox intervention. J Pharmacol Exp Ther 2011;336:56-63.

-29 Siitonen T, Siitonen P, Kinnula V, Mantymaa P, Savolainen ER, Koistinen P: Contribution of the induction of heme oxygenase-1 to etoposide-induced apoptosis in acute myeloblastic leukemia. Haematologica 2003;88:112-113.

-30 Jiang Y, Chen L, Tang Y, Ma G, Shen C, Qi C, Zhu Q, Yao Y, Liu N: HO-1 gene overexpression enhances the beneficial effects of superparamagnetic iron oxide labeled bone marrow stromal cells transplantation in swine hearts underwent ischemia/reperfusion: an MRI study. Basic Res Cardiol 2010;105:431-442.

-31 Yeh CH, Chen TP, Wang YC, Lin YM, Lin PJ: HO-1 activation can attenuate cardiomyocytic apoptosis via inhibition of NF- $\mathrm{KB}$ and AP-1 translocation following cardiac global ischemia and reperfusion. J Surg Res 2009;155:147-156.

- 32 Chen C, Huo R, Tong Y, Sheng Y, Liu HB, Gao X, Nakajima O, Yang BF, Dong DL: Systemic heme oxygenase-1 transgenic overexpression aggravates pressure overload-induced cardiac hypertrophy in mice. Cell Physiol Biochem 2011;28:25-32.

-33 Traore HN, Meyer D: The effect of iron overload on in vitro HIV-1 infection. J Clin Virol 2004;31:S92-S98.

-34 Park SO, Kumar M, Gupta S: TGF-beta and iron differently alter HBV replication in human hepatocytes through TGF- $\beta$ /BMP signaling and cellular microRNA expression. PLoS One 2012; 7:e39276. 